\title{
Atomic data of tungsten for current and future uses in fusion and plasma science
}

J. Clementson, P. Beiersdorfer, T. Lennartsson

August 6, 2012

22nd International Conference on the Application of Accelerators in Research and Industry

Fort Worth, TX, United States

August 5, 2012 through August 10, 2012 
This document was prepared as an account of work sponsored by an agency of the United States government. Neither the United States government nor Lawrence Livermore National Security, LLC, nor any of their employees makes any warranty, expressed or implied, or assumes any legal liability or responsibility for the accuracy, completeness, or usefulness of any information, apparatus, product, or process disclosed, or represents that its use would not infringe privately owned rights. Reference herein to any specific commercial product, process, or service by trade name, trademark, manufacturer, or otherwise does not necessarily constitute or imply its endorsement, recommendation, or favoring by the United States government or Lawrence Livermore National Security, LLC. The views and opinions of authors expressed herein do not necessarily state or reflect those of the United States government or Lawrence Livermore National Security, LLC, and shall not be used for advertising or product endorsement purposes. 
Atomic Data of Tungsten for Current and Future Uses in Fusion and Plasma Science physics. For example, accurate knowledge of atomic properties has been crucial for understanding the plasma energy balance and for diagnostic development. With the shift in magnetic fusion research toward hightemperature burning plasmas like those expected to be found in the ITER tokamak, the atomic physics of tungsten has become of importance. Tungsten will be a constituent of ITER plasmas because of its use as a

10 plasma-facing material able to withstand high heat loads with lower tritium retention than other possible

11 materials. Already, ITER diagnostics are being developed based on using tungsten radiation. In particular, 12 the ITER Core Imaging X-ray Spectrometer (CIXS), which is designed to measure the core ion temperature 13 and bulk plasma motion, is being based on the $\mathrm{x}$-ray emission of neonlike tungsten ions $\left(\mathrm{W}^{64+}\right)$. In addition, 14 tungsten emission will at ITER be measured by extreme ultraviolet (EUV) and optical spectrometers to 15 determine its concentration in the plasma and to assess power loss and tungsten sputtering rates. On present16 day tokamaks tungsten measurements are therefore being performed in preparation of ITER. Tungsten has 17 very complex spectra and most are still unknown. The WOLFRAM project at Livermore aims to produce 18 data for tungsten in various spectra bands: L-shell x-ray emission for CIXS development, soft x-ray and EUV 19 M- and N-shell tungsten emission for understanding the edge radiation from ITER plasmas and 20 contemporary tokamaks, and O-shell emission for developing spectral diagnostics of the ITER divertor.

21 Keywords: spectroscopic plasma diagnostics, atomic data, tungsten, tokamak, ITER, EBIT

\footnotetext{
${ }^{1}$ Present address:
} 


\section{INTRODUCTION}

Accurate atomic data on tungsten ions $(Z=74)$ have gained importance in the last few years as tungsten research

3 has become frequent at magnetic fusion facilities. The interest in tungsten spectra is spurred by the design choice of

4 the heavy element as a plasma-facing material in the ITER divertor [1] due to its favorable physical and chemical

5 properties: it has a high melting point, low sputtering yields, high-energy sputtering threshold, and low tritium

6 retention. On the agenda for several present-day fusion machines is to operate with tungsten components to learn

7 machine behavior and study the effects of tungsten-seeded plasmas. Especially the JET tokamak is pursuing this

8 with the recent installation of the ITER-like wall [2] and the ASDEX Upgrade tokamak that for many years has been

9 the leading experiment for tungsten tokamak studies [3]. Tungsten injection experiments at several other magnetic

10 fusion facilities have focused on the spectral signatures or particle transport, see e.g. [4, 5, 6]

11 Tungsten spectroscopy has several applications for a fusion plasma: it provides assessments of the tungsten

12 influx to the plasma (and thus of the wall material sputtering rates and particle transport), the plasma power balance,

13 and for diagnostics of plasma parameters. With tungsten distributed over the ITER plasma volume ions of various

14 charge states allow for measurements of the local plasma conditions.

15 Several studies of tungsten spectra have been performed at tokamaks and other magnetically confined fusion

16 plasmas, but the overwhelming majority of recent spectroscopic investigations of tungsten ions has been performed

17 on electron beam ion traps (EBITs), notably the EBIT laboratories at Berlin (now decommisioned), NIST, Tokyo,

18 and Livermore. Among these measurements are radiative and dielectronic recombination studies of L-shell $\mathrm{W}$ ions

19 by Biedermann et al. [7], and Watanabe et al. [8]; M1 transitions in M-shell W ions by Ralchenko et al. [9]; optical

20 transitions in highly charged W ions by Utter et al. [10], Komatsu et al. [11] and Watanabe et al. [12]; and intrashell

$21 \mathrm{x}$-ray transitions in L-shell W ions by Podpaly et al. [13]. At the Livermore EBIT facility the WOLFRAM project

22 addresses the atomic data need on tungsten ions for fusion plasma diagnostics [14]. High-precision and spectral

23 survey measurements are performed on the EBIT-I and SuperEBIT electron beam ion traps and complemented with

24 measurements on fusion plasma experiments, such as the Livermore SSPX spheromak, the Princeton NSTX

25 spherical torus, and the MIT Alcator C-Mod tokamak. Theoretical and modeling work are done in collaboration with

26 groups at Livermore, the University of Nevada at Reno, and Lund University. 


\section{ATOMIC DATA FOR THE ITER CORE IMAGING X-RAY SPECTROMETER}

The ion-temperature, $T_{i}$, and poloidal and toroidal rotation velocity, $v_{\phi}$ and $v_{\theta}$, profiles of the ITER core plasmas

3 will be measured using the Core Imaging X-ray Spectrometer (CIXS) [15]. The instrument is being designed for

4 Doppler measurements of the L-shell spectra of highly charged tungsten ions. Centered on the spectrum of Ne-like

5 W LXV, the $n=2-3$ transitions fall in the $8-12 \mathrm{keV}(1.0-1.5 \AA)$ x-ray interval where the high-resolution crystal

6 spectrometer will focus on one or a few spectral lines for measurements of line profiles and shifts. The CIXS may

7 also include a broadband moderate-resolution x-ray calorimeter to facilitate diagnostics of the ITER core electron

8 temperatures, $T_{e}$, and ion impurities [16]. To interpret the spectra and take full advantage of the diagnostic

9 capabilities of the CIXS, accurate radiative and collisional data for W L- and M-shell ions are required [17]. Several

10 spectroscopic studies on highly charged tungsten ions applicable to CIXS have been carried out using EBIT

11 spectroscopy $[7,8,18,19,20]$.

12 Tungsten has been chosen as the medium to probe the parameters of the ITER core plasmas since it will exist as

13 an indigenous impurity element in ITER plasmas providing strong x-ray emission over a large electron-temperature

14 interval. This is especially true for Ne-like $\mathrm{W}^{64+}$, which, due to its closed-shell structure, has a fractional abundance 15 of more than $10 \%$ between 12 and $33 \mathrm{keV}$. Mid- $Z$ elements predicted to be found in ITER plasmas, such as $\operatorname{Ar}(Z=$

16 18), $\mathrm{Fe}(Z=26)$, and $\mathrm{Cu}(Z=29)$, will mostly be fully stripped in the core plasmas, with expected electron

17 temperatures between 20 and $40 \mathrm{keV}$. An earlier design of the CIXS instrument was therefore open to the possibility

18 of introducing $\operatorname{Kr}(Z=36)$ into the tokamak for measurements of the He-like $\mathrm{Kr}$ XXXV spectrum [21]. To compare

19 the strengths of the Ka emissions from He-like Kr XXXV to the L-shell transitions in Ne-like W LXV, the spectra

20 have been calculated using the Flexible Atomic Code, FAC v.1.1.1 [22, 23] and modeled for steady-state ITER

21 plasma conditions, see Table 1. The diagnostically interesting tungsten transitions have much higher emissivities

22 than the krypton transitions ${ }^{2}$. However, it is important to note that the total ionization energy of almost $140 \mathrm{keV}$

23 required to create a Ne-like $\mathrm{W}^{64+}$ ion [25] is about 3.5 times higher than the roughly $40 \mathrm{keV}$ needed to make a He-

24 like $\mathrm{Kr}^{34+}$ ion. Whereas tungsten is expected to be an intrinsic impurity in ITER plasmas, krypton would need to be

25 injected. Even if tungsten would not exist in quantities sufficient to provide enough spectral signal, less tungsten

26 than krypton will need to be introduced due to the much higher line emissivities. For a given signal strength, the

27 energy consumed by $\mathrm{W}$ and $\mathrm{Kr}$ contribute about the same to the tokamak power balance (excluding bremsstrahlung). 
1 This, of course, also strongly depends on the charge state distributions of tungsten and krypton ions and on the

2 transport of the ions from the edge to the core plasmas ${ }^{3}$.

3 In order to accurately infer the ion temperatures, $T_{i}$, and bulk rotational velocities, $v_{\phi}$ and $v_{\phi}$, of the ITER plasmas

4 from the Doppler effect, the rest line positions and shapes (strengths and widths) of the $\mathrm{x}$-ray transitions must be

5 well known from laboratory measurements. The very-high ion-temperatures in the core plasmas will broaden the

6 lines to several electronvolts. Due to the high atomic mass of W, the Doppler widths of the $\mathrm{x}$ rays will be narrower

7 for $\mathrm{W}$ than for $\mathrm{Kr}$ and mid-Z ions. Table 2 lists natural line widths together with Doppler widths of He-like Kr XXXV

8 and Ne-like W LXV for temperatures of $T_{e}=T_{i}=10,20,30$, and $40 \mathrm{keV}$. Many of the listed transitions have very

9 short upper level lifetimes, in particular $\mathrm{Kr}$ XXXV Ka w with 640 as and W LXV 3D with 350 as, giving rise to line

10 broadenings of about 0.5 and $0.9 \mathrm{eV}$, respectively. For a typical CIXS spectrometer resolving power $R=E / \Delta E$ less

11 than about 14000 it does not matter that $\mathrm{Kr}$ has narrower natural line widths than $\mathrm{W}$. Conversely, for a spectrometer

12 with a resolution close to, or below, the natural width of $\mathrm{W} 3 \mathrm{D}$ it is essential to know the exact line shape.

13 Spectral surveys and detailed measurements of W L-shell transitions have been performed at the Livermore

14 EBIT facility $[18,19,20]$. Using a high-resolution crystal spectrometer in the von Hámos geometry at EBIT-I all the

15 strong $n=2-3$ lines in the Al-like W LXIII through O-like W LXVII spectra could be measured with high accuracy

16 [20], see Fig. 1 for the Ne-like W LXV spectrum. Employing one of the x-ray calorimeter spectrometers from the

17 Livermore-NASA laboratory astrophysics program [27], moderate-resolution spectra of W L-shell ions have been

18 acquired at SuperEBIT for several electron excitation energies [19]. Shown in Fig. 1 is the $8-10 \mathrm{keV}$ spectral

19 region at $E_{\text {beam }}=23.5 \mathrm{keV}$ where Ne-like W LXV dominates. Although the x-ray calorimeter may be operated to

20 attain line widths below $5 \mathrm{eV}$ at lower $\mathrm{x}$-ray energies, the energy resolution achieved for this measurement was

21 around $11 \mathrm{eV}$. The rapid development of $\mathrm{x}$-ray calorimeters will likely result in energy resolutions of $\sim 2 \mathrm{eV}$ in the

22 near future, making the addition of such an instrument to the CIXS diagnostic a practical option for broadband core

23 impurity measurements [16].

24 The CIXS instrument design focuses on the Ne-like W LXV spectrum, in particular the 3D line, but, depending

25 on the final spectrometer geometry and crystal selection, transitions from neighboring tungsten spectra may also be

26 covered. This would then also allow for the core tungsten charge balance to be measured. Using FAC, collisional-

27 radiative modeling of the $\mathrm{W}$ L-shell spectra have been performed to study how they develop with electron and ion

\footnotetext{
${ }^{2}$ It is furthermore easier to find crystals with appropriate $2 \mathrm{~d}$ spacings and high reflectivities for W L-shell $\mathrm{x}$ rays than for Kr K-shell $\mathrm{x}$ rays.
} 
1 temperatures. Figure 2 shows the calculated Ar-like W LVII through Li-like W LXXII spectra in the $8-10 \mathrm{keV}$ interval for $T_{e}=T_{i}=10$ and $30 \mathrm{keV}$.

\section{ATOMIC DATA OF TUNGSTEN FOR EDGE AND OHMIC CORE PLASMAS}

In plasmas of temperatures from a few hundred electronvolts to a few $\mathrm{keV}$ tungsten emission will be dominated by M- and N-shell transitions, mainly in the EUV and soft-x-ray parts of the spectrum. Since tungsten was first observed in tokamak plasmas in the mid-1970s by Isler et al. at the ORMAK tokamak [28] and by Hinnov et al. at the PLT tokamak [29], the strong quasicontinuum around $50 \AA$ has been found in essentially all magnetically confined plasmas with tungsten impurity contents. Although this emission is extremely bright, the spectral

9 complexity has prevented detailed diagnostic information to be extracted from it. The emission arises from $\Delta n=0$

$10 \mathrm{~N}$-shell transitions from a multitude of $\mathrm{N}$-shell tungsten charge states with transitions very close in energy. EBIT

11 measurements at Berlin [30] and Livermore [31] have stepped through the tungsten charge states by varying the

12 electron-beam energy and observing the resulting EUV emission. More detailed high-resolution measurements may

13 discover useful diagnostic lines, something that could be very beneficial given the extremely strong emission over a

14 wide electron-temperature range.

15 In $\mathrm{keV}$-plasmas the $\mathrm{W} \mathrm{M}$-shell ions dominate the charge balance. The $n=3-4$ spectra have been observed in 16 ASDEX Upgrade plasmas [32], and EBIT experiments at NIST [33] and Livermore [34, 35, 36, 37] have measured

17 many transitions in high resolution, especially in charge states near the closed-shell Ni-like $\mathrm{W}^{46+}$ ion. Figure 3 shows

18 a measured spectrum from the Livermore EBIT-I at an electron-beam energy of $6.5 \mathrm{keV}$ acquired with an $\mathrm{x}$-ray 19 calorimeter spectrometer with an energy resolution of $4.5 \mathrm{eV}$. The lines are identified by spectral modeling 20 calculations and recognized as $n=3-4$ transitions in Ge-like $\mathrm{W}^{42+}$ through Ti-like $\mathrm{W}^{52+}[14,19]$.

21 Tungsten ions with $3 \mathrm{~s}$ or $3 \mathrm{p}$ valence electrons have been studied in EBIT experiments at NIST [38, 39] and 22 Livermore [40, 41]. The $\mathrm{W}$ ions isoelectronic to $\mathrm{Na}, \mathrm{W}^{63+}$, and $\mathrm{Mg}, \mathrm{W}^{62+}$, have $\Delta n=0$ emission that should be 23 strong also at core plasma temperatures. From these relatively simple spectra the complexity quickly develops going 24 to lower charge states with more electrons, making the structure and spectra of most $\mathrm{W}$ ions with $3 \mathrm{p}$ and $3 \mathrm{~d}$ valence 25 electrons challenging to model accurately. Recently, $\Delta n=0$ transitions in $\mathrm{W}^{48+}-\mathrm{W}^{61+}$ in the $27-40 \AA$ interval were 26 measured at the Livermore EBIT-I to provide benchmark data for electron-correlation calculations [41].

\footnotetext{
${ }^{3}$ The radiative cooling rates for a given charge balance of Kr have been studied at the Berlin EBIT facility [26].
} 


\section{ATOMIC DATA OF TUNGSTEN FOR ITER DIVERTOR DIAGNOSTICS}

It will be important to monitor the concentration of tungsten ions in ITER divertor plasmas in order to infer the sputtering rates from the plasma-facing components. Furthermore, the large abundance of tungsten ions may also be applied for charge-balance, electron-temperature, and electron-density diagnostics. ITER divertor plasmas will be sufficiently cool ( $T_{e}$ around $1 \mathrm{eV}$ close to the target plates and up to $150 \mathrm{eV}$ near the $\mathrm{X}$ point) and dense $\left(N_{e} \sim 10^{15}\right.$ $\mathrm{cm}^{-3}$ ) so that only few-times charged tungsten ions will have large fractional abundances in thermodynamic equilibrium, cf. [42]. Only the spectra of the first few charge states have been studied $[43,44]$ and most of these investigations have been at electron densities much higher than magnetic fusion densities so that the spectra may not resemble the emission from tokamak divertors. Experiments to simulate this emission have been undertaken at the MT-1M tokamak [45], the Livermore SSPX spheromak [46], the Institute of Spectroscopy at the Russian Academy

11 of Sciences and the Meudon Observatory [47], and, recently also at the Livermore EBIT facility.

12 The Sustained Spheromak Physics Experiment (SSPX) was the latest magnetic confinement experiment at 13 Livermore, in operation from 1999 to 2007. The primary goals of the project was to investigate magnetic field build 14 up and to evaluate the spheromak as a fusion reactor concept [48]. With modest electron temperatures (typically 15 below $200 \mathrm{eV}$ ) and densities in the $10^{14-15} \mathrm{~cm}^{-3}$ range SSPX also provided a very suitable testbed for spectroscopic 16 studies relevant to divertor plasmas of large tokamaks. Tungsten injection experiments provided enhancements to 17 the intrinsic tungsten concentration (originating from the flux conserver coating) and aided the analysis of tungsten 18 EUV spectra. Shown in Fig. 5 is a spectrum [46] acquired with a grazing-incidence instrument [49] at SSPX during 19 injection of tungsten hexacarbonyl, $\mathrm{W}(\mathrm{CO})_{6}$. As a result, both $\mathrm{W}$ and $\mathrm{O}$ line emission were enhanced. The strongest 20 W emission is from Er-like W VII, which could be identified (though the line intensities do not agree) based on the 21 sliding-spark measurements of Sugar and Kaufman [50]. The remaining emission attributed to tungsten is believed 22 to belong to the neighboring charge states, but the complexity and modest spectral resolution have prevented 23 definitive line identifications.

24 A more systematic study of the EUV spectra from few-times ionized tungsten have therefore been performed 25 using EBIT spectroscopy. Operating the Livermore EBIT-I at low voltages, the excitation of tungsten spectra could 26 be varied in the $30-300 \mathrm{eV}$ range. Spectra in the $120-320 \AA$ were acquired using a $5.6 \mathrm{~m} 1200$ lines/mm grazing27 incidence spectrometer (similar to the instrument used at SSPX) equipped with a Princeton Instruments CCD 28 detector. High-resolution data of mainly Tm-like W VI, Er-like W VII, and Ho-like W VIII were obtained using a 
$144.3 \mathrm{~m}$ grating spectrometer [51]. Figure 5 illustrates the advantages of scanning the electron-beam energy for interpretation of plasma-produced spectra where the SSPX spectrum is followed by two panels with EBIT tungsten data at $E_{\text {beam }}=135$ and $163 \mathrm{eV}$.

\section{SUMMARY}

Recent years' atomic spectroscopy efforts of tungsten ions have produced much atomic data important for powerbalance estimates and diagnostics of magnetically confined plasmas. Still, since tungsten impurity ions in future plasma devices can be expected to be found both in cooler regions, such as divertor and near-wall plasmas, as well as in moderate and hot regions, such as edge and core plasmas, there is a significant need for additional radiative and

9 collisional data on tungsten in essentially all ionization stages. Presented in this paper is an overview of recent 10 results from the Livermore WOLFRAM project, which has produced data on L- and M-shell W ions important for 11 the ITER CIXS instrument, M-shell $\mathrm{W}$ ions for ITER edge and present-day plasmas, and O-shell W ions for ITER 12 divertor diagnostics.

\section{ACKNOWLEDGMENTS}

14 Work was performed at Lawrence Livermore National Laboratory under the auspices of the U.S. Department of 15 Energy under contract DE-AC52-07NA27344 and carried out as part of the International Atomic Energy Agency 16 Coordinated Research Project Spectroscopic and Collisional Data for Tungsten from $1 \mathrm{eV}$ to $20 \mathrm{keV}$. The authors 17 wish to acknowledge support from Dr. M. F. Gu and Mr. E. W. Magee.

\section{REFERENCES}

1. R. A. Pitts, A. Kukushkin, A. Loarte, A. Martin, M. Merola, C. E. Kessel, V. Komarov, and M. Shimada, Phys. Scr. T138, 014001 (2009)

22 2. G. F. Matthews, M. Beurskens, S. Brezinsek, M. Groth, E. Joffrin, A. Loving, M. Kear, M.-L. Mayoral, R. Neu, 23 P. Prior, V. Riccardo, F. Rimini, M. Rubel, G. Sips, E. Villedieu, P. de Vries, M. L. Watkins, and EFDA-JET contributors, Phys. Scr. T145, 014001 (2011) 
3. R. Neu, V. Bobkov, R. Dux, J. C. Fuchs, O. Gruber, A. Herrmann, A. Kallenbach, H. Maier, M. Mayer, T. Pütterich, V. Rohde, A. C. C. Sips, J. Stober, K. Sugiyama, and ASDE Upgrade Team, Phys. Scr. T138, 014038 (2009)

4. M. B. Chowdhuri, S. Morita, M. Goto, H. Nishimura, K. Nagai, and S. Fujioka, Plasma Fusion Res. 2, S1060 (2007)

5. C. S. Harte, C. Suzuki, T. Kato, H. A. Sakaue, D. Kato, K. Sato, N. Tamura, S. Sudo, R. D'Arcy, E. Sokell, J. White, and G. O'Sullivan, J. Phys. B: At. Mol. Opt. Phys. 43, 205004 (2010)

6. J. Clementson, P. Beiersdorfer, A. L. Roquemore, C. H. Skinner, D. K. Mansfield, K. Hartzfeld, and J. K. Lepson, Rev. Sci. Instrum. 81, 10E326 (2010)

7. C. Biedermann, R. Radtke, R. Seidel, and T. Pütterich, Phys. Scr. T134, 014026 (2009)

11 8. H. Watanabe, N. Nakamura, D. Kato, T. Nakano, and S. Ohtani, Plasma Fusion Res. 2, 027 (2007)

12 9. Yu. Ralchenko, I. Draganić, D. Osin, J. D. Gillaspy, and J. Reader, Phys. Rev. A 83, 032517 (2011)

13 10. S. B. Utter, P. Beiersdorfer, and G. V. Brown, Phys. Rev. A 61, 030503(R) (2000)

14 11. A. Komatsu, J. Sakoda, N. Nakamura, H. A. Sakaue, X.-B. Ding, D. Kato, I. Murakami, and F. Koike, Phys. Scr. 15 T144, $014012(2011)$

16 12.H. Watanabe, N. Nakamura, D. Kato, H. A. Sakaue, S. Ohtani, Can. J. Phys. 90, 497 (2012)

17 13. Y. Podpaly, J. Clementson, P. Beiersdorfer, J. Williamson, G. V. Brown, and M. F. Gu, Phys. Rev. A 80, 052504 18 (2009)

19 14. J. Clementson, P. Beiersdorfer, G. V. Brown, M. F. Gu, H. Lundberg, Y. Podpaly, and E. Träbert, Can. J. Phys. $20 \quad \mathbf{8 9}, 571(2011)$

21 15. P. Beiersdorfer, J. Clementson, J. Dunn, M. F. Gu, K. Morris, Y. Podpaly, E. Wang, M. Bitter, R. Feder, K. W. 22 Hill, D. Johnson, and R. Barnsley, J. Phys. B: At. Mol. Opt. Phys. 43, 144008 (2010)

23 16. P. Beiersdorfer, G. V. Brown, J. Clementson, J. Dunn, K. Morris, E. Wang, R. L. Kelley, C. A. Kilbourne, F. S. Porter, M. Bitter, R. Feder, K. W. Hill, D. Johnson, and R. Barnsley, Rev. Sci. Instrum. 81, 10E323 (2010)

17.J. Clementson, P. Beiersdorfer, C. Biedermann, M. Bitter, L. F. Delgado-Aparicio, A. Graf, M. F. Gu, K. W. Hill, and R. Barnsley, Europhys. Conf. Abs. 36, P2.040 (2012)

27 18.P. Beiersdorfer, AIP Conf. Proc. 274, 365 (1993)

28 19. J. Clementson, Ph.D. Dissertation, Lund University (2010) 
1 20. P. Beiersdorfer, J. K. Lepson, M. B. Schneider, and M. P. Bode, Phys. Rev. A 86, 012509 (2012)

2 21. R. Barnsley, M. O'Mullane, L. C. Ingesson, and A. Malaquias, Rev. Sci. Instrum. 75, 3743 (2004)

3 22. M. F. Gu, AIP Conf. Proc. 730, 127 (2004)

4 23. M. F. Gu, Can. J. Phys. 86675 (2008)

5 24. K. Widmann, P. Beiersdorfer, V. Decaux, and M. Bitter, Phys. Rev. A 53, 2200 (1996)

6 25. P. Beiersdorfer, M. J. May, J. H. Scofield, and S. B. Hansen, High Energy Density Phys. 8, 271 (2012)

7 26. R. Radtke, C. Biedermann, T. Fuchs, G. Fußmann, and P. Beiersdorfer, Phys. Rev. E 61, 1966 (2000)

8 27.F. S. Porter, J. S. Adams, P. Beiersdorfer, G. V. Brown, J. Clementson, M. Frankel, S. M. Kahn, R. L. Kelley,

9 and C. A. Kilbourne, AIP Conf. Proc. 1185, 454 (2009)

10 28. R. C. Isler, R. V. Neidigh, and R. D. Cowan, Phys. Lett. 63 A, 295 (1977)

11 29. E. Hinnov and M. Mattioli, Phys. Lett. 66 A, 109 (1978)

12 30. R. Radtke, C. Biedermann, J. L. Schwob, P. Mandelbaum, and R. Doron, Phys. Rev. A 64, 012720 (2001)

13 31. S. B. Utter, P. Beiersdorfer, and E. Träbert, Can. J. Phys. 80, 1503 (2002)

14 32. R. Neu, K. B. Fournier, D. Schlögl, and J. Rice, J. Phys. B: At. Mol. Opt. Phys. 30, 5057 (1997)

15 33. Yu. Ralchenko, J. N. Tan, J. D. Gillaspy, J. M. Pomeroy, and E. Silver, Phys. Rev. A 74, 042514 (2006)

16 34.P. Neill, C. Harris, A. S. Safronova, S. Hamasha, S. Hansen, U. I. Safronova, and P. Beiersdorfer, Can. J. Phys.

$17 \mathbf{8 2}, 931(2004)$

18 35. J. Clementson, P. Beiersdorfer, G. V. Brown, and M. F. Gu, Phys. Scr. 81, 015301 (2010)

19 36. J. Clementson, P. Beiersdorfer, and M. F. Gu, Phys. Rev. A 81, 012505 (2010)

20 37. C. G. Osborne, A. S. Safronova, V. L. Kantsyrev, U. I. Safronova, P. Beiersdorfer, K. M. Williamson, M. E. 21 Weller, and I. Shresta, Can. J. Phys. 89, 599 (2011)

22 38. Yu. Ralchenko, I. N. Draganic, J. N. Tan, J. D. Gillaspy, J. M. Pomeroy, J. Reader, U. Feldman, and G. E. 23 Holland, J. Phys. B: At. Mol. Opt. Phys. 41, 021003 (2008)

24 39. J. D. Gillaspy, I. N. Draganić, Yu. Ralchenko, J. Reader, J. N. Tan, J. M. Pomeroy, and S. M. Brewer, Phys. Rev. $25 \quad A \mathbf{8 0}, 010501(\mathrm{R})(2009)$

26 40. J. Clementson and P. Beiersdorfer, Phys. Rev. A 81, 052509 (2010)

27 41. T. Lennartsson, J. Clementson, and P. Beiersdorfer, Phys. Rev. A, in preparation (2012)

28 42. A. E. Kramida and J. Reader, At. Data Nucl. Data Tables 92, 457 (2006) 
1 43. A. E. Kramida and T. Shirai, At. Data Nucl. Data Tables 95, 305 (2009)

2 44. A. Kramida, Can. J. Phys. 89, 551 (2011)

3 45. G. Veres, J. S. Bakos, and B. Kardon, J. Quant. Spectrosc. Radiat. Transfer 56, 295 (1996)

4 46. J. Clementson, P. Beiersdorfer, E. W. Magee, H. S. McLean, R. D. Wood, J. Phys. B: At. Mol. Opt. Phys. 43, $5 \quad 144009(2010)$

6 47. W.-Ü. L. Tchang-Brillet, J.-F. Wyart, A. N. Ryabtsev, R. R. Kildiyarova, and E. Ya. Kononov, ASOS 10 Book of 7 Abstracts, Berkeley, California (2010)

8 48. B. Hudson, R. D. Wood, H. S. McLean, E. B. Hooper, D. N. Hill, J. Jayakumar, J. Moller, D. Montez, C. A. 9 Romero-Talamás, T. A. Casper, J. A. Johnson III, L. L. DoDestro, E. Mezonlin, and L. D. Pearlstein, Phys. 10 Plasmas 15, $056112(2008)$

11 49. J. Clementson, P. Beiersdorfer, and E. W. Magee, Rev. Sci. Instrum. 79, 10F538 (2008)

12 50. J. Sugar and V. Kaufman, Phys. Rev. A 12, 994 (1975)

13 51.P. Beiersdorfer, E. W. Magee, E. Träbert, H. Chen, J. K. Lepson, M.-F. Gu, and M. Schmidt, Rev. Sci. Instrum. $1475,3723(2004)$ 
TABLE 1. Predicted transition emissivities for electron-impact excitation in He-like Kr XXXV and Ne-like W LXV for plasmas with $N_{e}=10^{14} \mathrm{~cm}^{-3}$ and $T_{e}=10,20,30$, and $40 \mathrm{keV}$. Emissivities $\varepsilon$ are listed in units of photon per ion per second $\left(\gamma / Z^{q+} / \mathrm{s}\right)$. Experimental transition energies $\Delta E_{\text {exp }}$, are in units of electronvolt (eV).

\begin{tabular}{|c|c|c|c|c|c|}
\hline Line & $\Delta E_{\text {exp }}$ & $\varepsilon(10 \mathrm{keV})$ & $\varepsilon(20 \mathrm{keV})$ & $\varepsilon(30 \mathrm{keV})$ & $\varepsilon(40 \mathrm{keV})$ \\
\hline $\operatorname{Kr}$ XXXV K $\alpha w\left(1 s_{1 / 2}-2 p_{3 / 2}\right)$ & $13114.68(36)^{\mathrm{a}}$ & 45 & 82 & 101 & 111 \\
\hline $\operatorname{Kr} \mathrm{XXXV} \operatorname{K} \alpha \times\left(1 \mathrm{~s}_{1 / 2}-2 \mathrm{p}_{3 / 2}\right)$ & $13091.17(37)^{\mathrm{a}}$ & 7 & 7 & 6 & 5 \\
\hline $\operatorname{Kr} \operatorname{XXXV} K \alpha$ y $\left(1 s_{1 / 2}-2 p_{1 / 2}\right)$ & $13026.29(36)^{\mathrm{a}}$ & 16 & 26 & 30 & 32 \\
\hline $\operatorname{Kr} \operatorname{XXXV} \operatorname{Kaz}\left(1 s_{1 / 2}-2 s_{1 / 2}\right)$ & $12979.63(41)^{\mathrm{a}}$ & 8 & 9 & 9 & 8 \\
\hline $\mathrm{W} \operatorname{LXV} 3 \mathrm{~A}\left(2 \mathrm{~s}_{1 / 2}-3 \mathrm{p}_{3 / 2}\right)$ & $10706.85(90)^{\mathrm{b}}$ & 24 & 42 & 49 & 54 \\
\hline W LXV $3 C\left(2 p_{1 / 2}-3 d_{3 / 2}\right)$ & $10408.69(40)^{\mathrm{b}}$ & 146 & 217 & 244 & 257 \\
\hline $\mathrm{W} \operatorname{LXV} 3 \mathrm{~B}\left(2 \mathrm{~s}_{1 / 2}-3 \mathrm{p}_{1 / 2}\right)$ & $10317.23(50)^{b}$ & 48 & 76 & 87 & 93 \\
\hline W LXV $3 F\left(2 p_{1 / 2}-3 d_{3 / 2}\right)$ & $9689.29(50)^{\mathrm{b}}$ & 46 & 57 & 57 & 55 \\
\hline $\mathrm{W} \operatorname{LXV} 3 \mathrm{D}\left(2 \mathrm{p}_{3 / 2}-3 \mathrm{~d}_{5 / 2}\right)$ & $9126.25(50)^{\mathrm{b}}$ & 497 & 695 & 766 & 798 \\
\hline W LXV $3 E\left(2 p_{3 / 2}-3 d_{3 / 2}\right)$ & $8996.31(50)^{\mathrm{b}}$ & 27 & 32 & 33 & 32 \\
\hline $\mathrm{W}$ LXV $3 \mathrm{G}\left(2 \mathrm{p}_{3 / 2}-3 \mathrm{~s}_{1 / 2}\right)$ & $8307.51(40)^{\mathrm{b}}$ & 288 & 358 & 367 & 356 \\
\hline W LXV M2 $\left(2 \mathrm{p}_{3 / 2}-3 \mathrm{~s}_{1 / 2}\right)$ & $8299.22(40)^{\mathrm{b}}$ & 106 & 132 & 115 & 96 \\
\hline
\end{tabular}

$1 \quad{ }^{\mathrm{a}}$ Widmann et al. [24], ${ }^{\mathrm{b}}$ Beiersdorfer et al. [20] 
ID 56

TABLE 2. Calculated line widths for He-like Kr XXXV and Ne-like W LXV. $\boldsymbol{\Delta} \boldsymbol{E}_{\boldsymbol{N}}$ refers to natural broadening and $\boldsymbol{\Delta} \boldsymbol{E}_{\boldsymbol{D}}$ to Doppler broadening for $T_{i}=10,20,30$, and $40 \mathrm{keV}$. Units in electronvolt (eV).

\begin{tabular}{|c|c|c|c|c|c|}
\hline Line & $\Delta E_{N}$ & $\Delta E_{D}(10 \mathrm{keV})$ & $\Delta E_{D}(20 \mathrm{keV})$ & $\Delta E_{D}(30 \mathrm{keV})$ & $\Delta E_{D}(40 \mathrm{keV})$ \\
\hline $\operatorname{Kr} X X X V \operatorname{Kaw}\left(1 \mathrm{~s}_{1 / 2}-2 \mathrm{p}_{3 / 2}\right)$ & 0.52 & 7.44 & 10.53 & 12.89 & 14.89 \\
\hline $\operatorname{Kr} \operatorname{xxxV} \mathrm{K} \alpha \mathrm{x}\left(1 \mathrm{~s}_{1 / 2}-2 \mathrm{p}_{3 / 2}\right)$ & 0.00 & 7.43 & 10.51 & 12.87 & 14.86 \\
\hline $\operatorname{Kr} x x x V \operatorname{Kay}\left(1 \mathrm{~s}_{1 / 2}-2 \mathrm{p}_{1 / 2}\right)$ & 0.13 & 7.39 & 10.46 & 12.81 & 14.79 \\
\hline $\operatorname{Kr} x x x V \operatorname{Ka} z\left(1 s_{1 / 2}-2 s_{1 / 2}\right)$ & 0.00 & 7.37 & 10.42 & 12.76 & 14.73 \\
\hline $\mathrm{W} \operatorname{LXV} 3 \mathrm{~A}\left(2 \mathrm{~s}_{1 / 2}-3 \mathrm{p}_{3 / 2}\right)$ & 0.16 & 6.08 & 8.59 & 10.53 & 12.15 \\
\hline W LXV $3 C\left(2 p_{1 / 2}-3 d_{3 / 2}\right)$ & 0.49 & 5.91 & 8.35 & 10.23 & 11.81 \\
\hline $\mathrm{W} \operatorname{LXV} 3 \mathrm{~B}\left(2 \mathrm{~s}_{1 / 2}-3 \mathrm{p}_{1 / 2}\right)$ & 0.23 & 5.86 & 8.28 & 10.14 & 11.71 \\
\hline W LXV $3 F\left(2 p_{1 / 2}-3 d_{3 / 2}\right)$ & 0.01 & 5.50 & 7.77 & 9.52 & 10.99 \\
\hline $\mathrm{W} \operatorname{LXV} 3 \mathrm{D}\left(2 \mathrm{p}_{3 / 2}-3 \mathrm{~d}_{5 / 2}\right)$ & 0.95 & 5.18 & 7.32 & 8.97 & 10.35 \\
\hline W LXV $3 E\left(2 p_{3 / 2}-3 d_{3 / 2}\right)$ & 0.03 & 5.10 & 7.22 & 8.84 & 10.21 \\
\hline W LXV $3 G\left(2 p_{3 / 2}-3 s_{1 / 2}\right)$ & 0.05 & 4.71 & 6.66 & 8.16 & 9.43 \\
\hline W LXV M2 $\left(2 \mathrm{p}_{3 / 2}-3 \mathrm{~s}_{1 / 2}\right)$ & 0.00 & 4.71 & 6.66 & 8.15 & 9.42 \\
\hline
\end{tabular}


ID 56
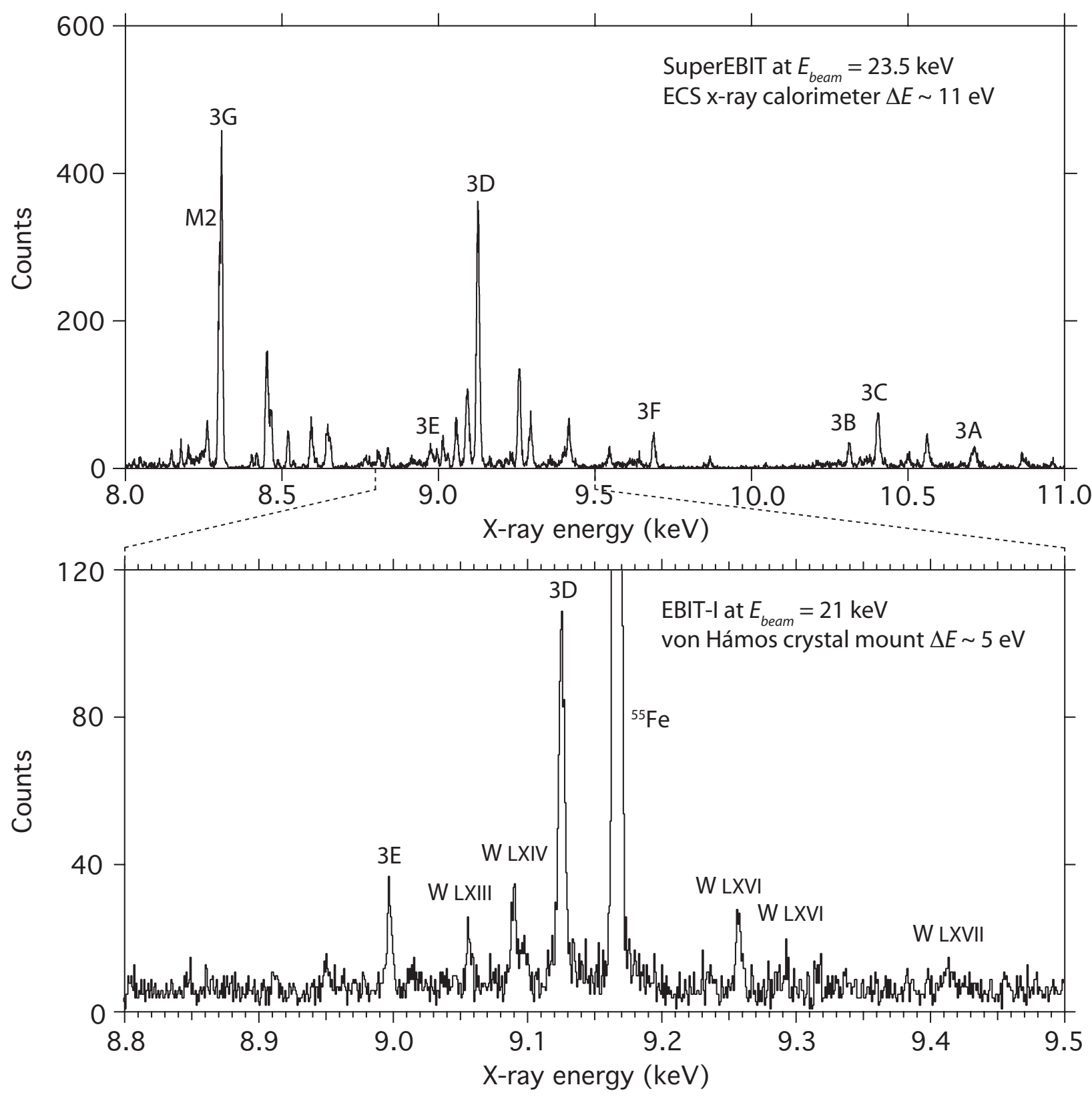

3 FIGURE 1. Ne-like W LXV spectra measured at the Livermore EBIT facility using a NASA x-ray calorimeter 4 spectrometer [19] and a von Hámos crystal spectrometer [20].

5

6 

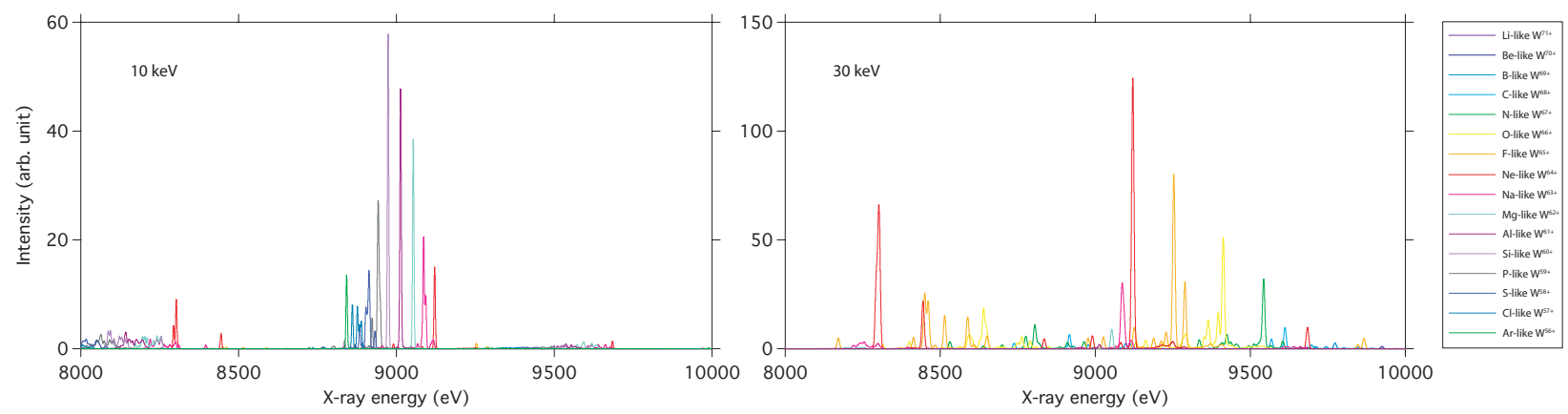

FIGURE 3. Theoretical spectra of W LVII - LXXII for $T_{e}=T_{i}=10$ and $30 \mathrm{keV}$ and $N_{e}=10^{14} \mathrm{~cm}^{-3}$.

300

Experiment
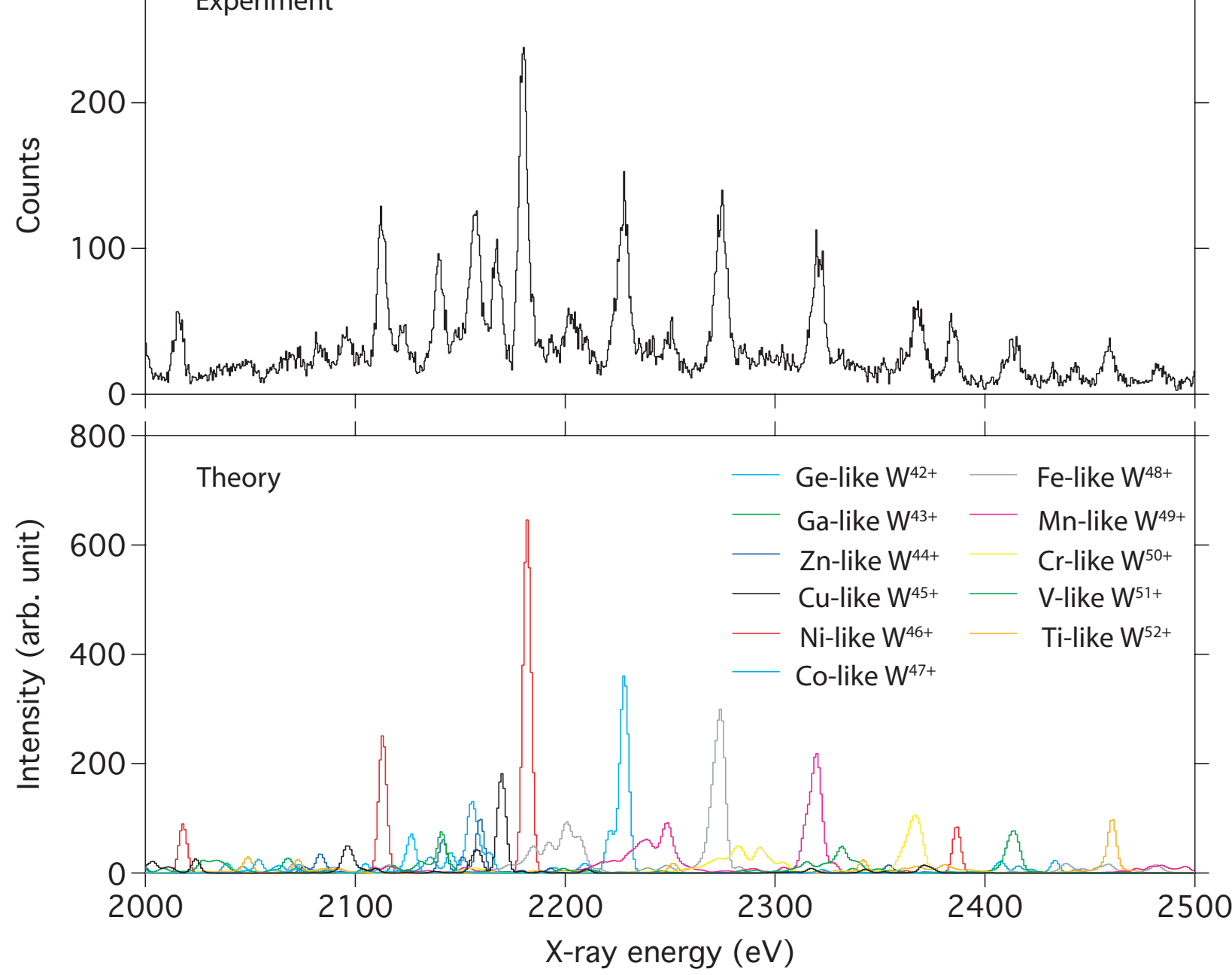

8 FIGURE 4. M-shell transitions from $\mathrm{M}$ - and $\mathrm{N}$-shell charge states of tungsten measured at the Livermore EBIT-

9 I for $E_{\text {beam }}=6.5 \mathrm{keV}$ and compared to collisional-radiative modeling [14, 19]. 


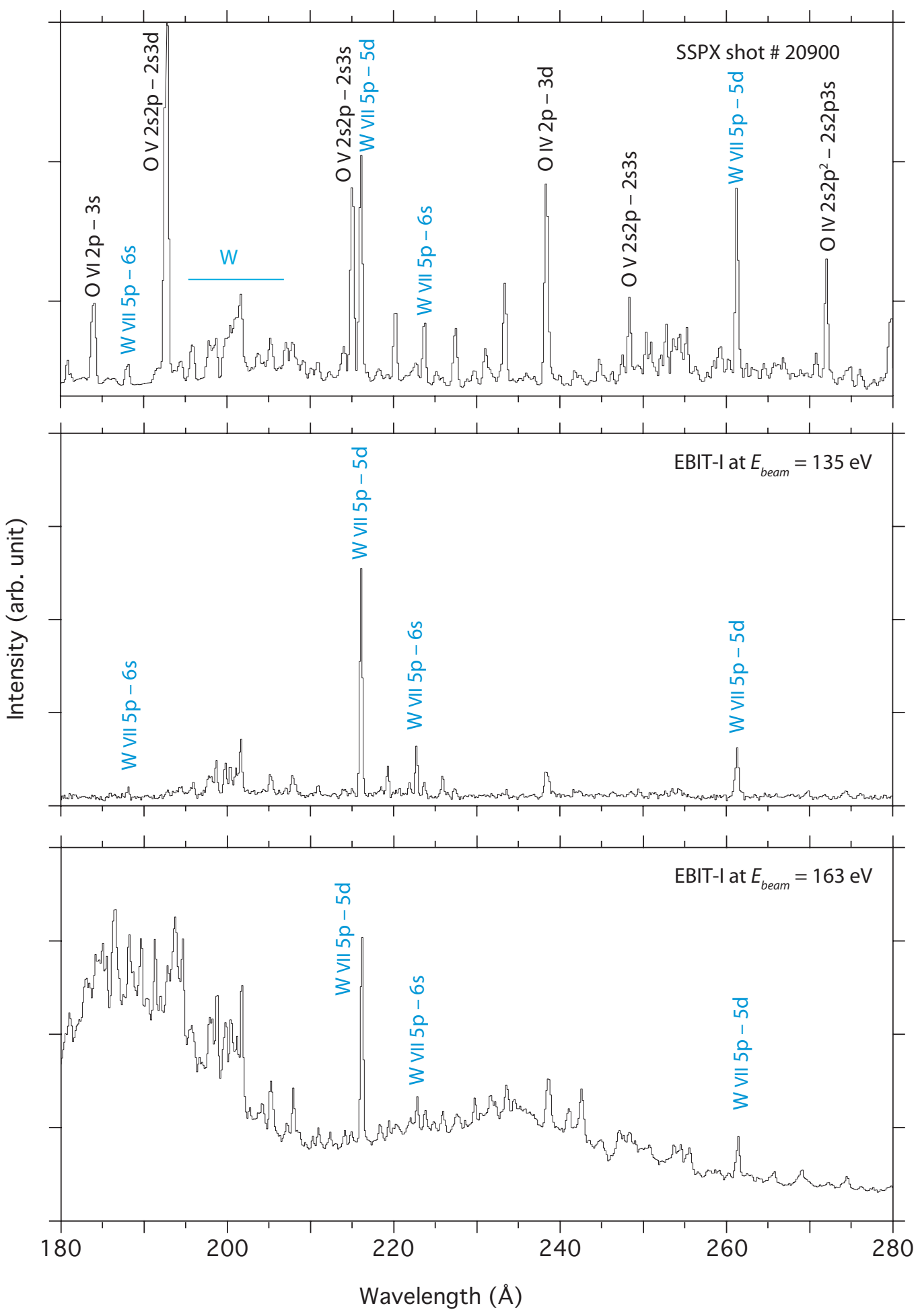

2

3 FIGURE 5. Spectra from low charge states of tungsten measured at the Livermore SSPX spheromak and the

4 Livermore EBIT-I electron beam ion trap. Lines not labeled in the SSPX spectrum are mainly from W, O, and Ti. 